Proceedings of the 2012 Winter Simulation Conference

C. Laroque, J. Himmelspach, R. Pasupathy, O. Rose, and A. M. Uhrmacher, eds.

\title{
SYMBIOTIC SIMULATION FOR FUTURE ELECTRO-MOBILITY TRANSPORTATION SYSTEMS
}

\author{
Heiko Aydt \\ TUM CREATE Ltd. \\ 62 Nanyang Drive \\ Singapore 637459
}

\author{
Michael Lees \\ School of Computer Engineering \\ Nanyang Technological University \\ Nanyang Avenue \\ Singapore 639798
}

\author{
Alois Knoll \\ Robotics and Embedded Systems Group \\ Department of Informatics \\ Technische Universität München \\ Boltzmannstraße 3 \\ D-85748 Garching bei München, GERMANY
}

\begin{abstract}
Electro-mobility is widely regarded as the future of transportation systems. The shift from fossil fuel-based engines to electro-mobility will pose new challenges to the operations of future transportation systems. Our vision of a smart transportation system for the future entails a collaborative communication and simulation infrastructure that can help to mitigate common traffic-related problems as well as problems that are specific to electric vehicles. At the core of this smart transportation system would be a symbiotic simulation system which incorporates information provided by the various traffic participants into city-scale traffic simulations. We describe the symbiotic simulation system and highlight the research challenges that need to be addressed in order to realize such a system. This includes a server-based city-scale simulation which would forecast general traffic patterns and conditions in the near-future. The outcome of these simulations can be used by server-based smart routing services and/or in-car navigation systems.
\end{abstract}

\section{INTRODUCTION}

The expectation of people, with regard to technology and convenience is increasing as fast as the technology itself. Everything is becoming smarter, whether it is smart cities (http://www-03.ibm.com/innovation/us/ thesmartercity/index_flash.html), smart buildings (Snoonian 2003), or smart transportation (Wang, Zeng, and Yang 2006), much of our surroundings are becoming more technologically sophisticated. The term smart can signify many things, each with different connotations of smartness. Generally, however, the smartness in question is a control system or some form of intelligent agent, which accepts input from sensors, makes sense of the information it collects, and then has some form of actuation on the environment which it is controlling. The sensors can be static physical sensors to measure environmental conditions (e.g., temperature) or mobile devices which sense information from people. For trivial examples of such systems the smart component can be realized in a straightforward manner, the control system may only need to satisfy a single objective with few constraints. However, the most interesting examples are generally composed of large numbers of interacting entities (humans), whose complex non-linear interactions lead 


\section{Aydt, Lees, and Knoll}

to hugely complex systems that create emergent dynamics which are often too complex to understand and control analytically.

Modeling and simulation is often employed as a means of trying to analyze, understand and control complex systems, including transportation systems. The application of modeling and simulation in the field of transportation dates back to the 1950s (Gerlough 1956). Simulation in this sense has been used very successfully as a tool for planning and building transportation infrastructure. The existing tools and methodologies within traffic engineering are not designed to capture real-time data and certainly not intended to provide real-time decision support to drivers. This is particularly true of Singapore, which has an extremely efficient (high throughput) but brittle (prone to high impact accidents) transportation network. Traffic is just one example of the types of human complex systems which could benefit from, what we term living simulations, a concept which provides a way to use simulation as means of real-time decision support and real-time decision-making.

We define Living Simulations as a form of symbiotic simulation, where the physical system contains some human component and provide information through participatory sensing. The central idea is to fuse ideas from collaborative, social computation with advanced individual-based modeling techniques to provide on-the-fly information support systems to individuals. The key motivation for the application of living simulations in the context of transportation systems, is to provide an intelligent traffic management system that can better manage the system and in doing so reduce the likelihood of failures, increase efficiency and increase robustness. Symbiotic simulation is a paradigm in which a physical system is closely coupled with a simulation system (Fujimoto et al. 2002; Aydt et al. 2008). The simulation system benefits from real-time sensor data about the physical system, i.e., sensor data is collected in real-time and used to perform high-fidelity simulations of the physical system. The physical system, on the other hand, benefits from the results of the simulation. For example, in the context of decision support, symbiotic simulation can be used to evaluate a (potentially) large amount of alternative decisions (what-if scenarios) by means of simulation before implementing them in the physical system.

Increasing the efficiency and robustness of traffic becomes even more important when considering electro-mobility. Some of the most crucial factors in the context of electro-mobility is the battery capacity and the resulting range of an electrical vehicle. Although research in battery technologies has made significant progress in recent years, it remains a critical issue. As of 2012, typical electrical vehicles such as the Mitsubishi I MiEV (Halvorson 2011) or the Mini E (Turrentine et al. 2011) have a range of approximately 130-180 kilometers. In practice the range depends much on the usage of the vehicle and its auxiliary components. In particular major energy consumers, such as air conditioning and heating systems, can have a significant impact on the overall energy consumption and thus also the range of the vehicle. Therefore, a smart transportation system would not only improve the efficiency of the transportation system and increase the throughput of traffic but also reduce the energy consumption of the individual traffic participants, thus improving the range of electrical vehicles and - more importantly - reducing the risk of break-downs due to out-of-energy incidents.

Transportation systems are highly complex due to the thousands of traffic participants and their interactions with each other. For example, in case of Singapore there are approximately 80k-90k vehicles driving on the road during peak hours. Typical traffic patterns, such as shock waves (Richards 1956), are emergent phenomena that result from the behavior of the drivers (e.g., lane changing, braking, and accelerating). Driver behavior is increasingly affected by technology. For example, many vehicles are now equipped with on-board navigation system and those which are not equipped with on-board systems can be easily upgraded with dedicated navigation systems (e.g., TomTom devices (http://www.tomtom.com/)) or by using smartphones and corresponding apps. These navigation systems are often also capable of incorporating traffic data that provides information about congestions, for example, in real-time. In principal all these navigation systems provide routing services to their users. While the routing itself can be arbitrarily sophisticated and also consider latest traffic information, all navigation systems essentially attempt to find the optimal route from one location to another. 


\section{Aydt, Lees, and Knoll}

Increasing use of such navigation systems can lead to interesting and sometimes bizarre incidents. For example, see this article (Rothman 2011) which highlights problems with GPS-based navigation systems. Often these issues are due to outdated or incomplete map material. However, even with accurate map material, existing navigation systems are not smart in the sense that they can forecast how the traffic situation will develop or anticipate how widespread use of navigation systems can cause problems of its own. For example, navigation systems can incorporate real-time traffic information and divert drivers in case of jams and slow traffic. However, if there are too many of those systems in use, it may cause a significant number of vehicles being diverted through the same alternative route and thus effectively diverting the congestion itself. In order to avoid such artifacts, it is necessary for navigation systems to be able coordinate their actions. A smart routing system will (at least partially) perform global optimization which keeps objectives regarding overall throughput and congestion minimization in mind while finding an individual's best route from one location to another.

Efficient transportation systems are crucial for today's mega-cities and will become even more important in the future which will be dominated by electric vehicles, posing new constraints and challenges but also new opportunities. In this paper, we explain how a smart routing service can be realized using the principles of symbiotic simulation. As mentioned above, smart routing requires coordinated actions and a sense for the global optimum. Such a smart routing system is one potential service that can leverage on a symbiotic simulation-based smart traffic system. In Section 2 we describe the general architecture of the system. In Section 3 we describe how a smart routing service can be realized. We present a list of research challenges in Section 4 and conclude the paper by presenting a summary in Section 5.

\section{SYSTEM DESIGN}

At the core of the smart traffic system is a high-fidelity simulation model of the entire city. In order to achieve the required degree of fidelity, it is necessary to incorporate various kinds of data from different sources. In general, it can be distinguished between historical data and real-time data. Such data can include map data, which contains information about the road network, intersections, as well as locations of traffic lights; traffic pattern data, which is typically historical and provides information regarding tempo-spatial traffic flow patterns (e.g., average speed at a certain time of the day on a particular road link, throughput in terms of a number of vehicles per time period, etc.); operational data which provides information regarding how the infrastructure (in particular traffic lights) is operated (e.g., signal timings); live traffic data from various sensors (e.g., cameras) which provide information regarding the current traffic situation (e.g., average speed, throughput, traffic density, etc.); and real-time data from various traffic participants which provide information such as the position via mobile phones, for example. In general, the more data that can be incorporated into the simulation model, the higher the fidelity of the model. However, aggregating vast amounts of data is challenging. In addition, some of the required data is not available at design time (e.g., live traffic data) and has to be incorporated during run-time.

Even without using the data for simulation purposes, the ability to aggregate and visualize data from various sources in real-time can already represent a useful functionality for analyzing the current traffic situation. A first step toward a smart traffic system would be to provide an appropriate real-time visualization of the current traffic situation to traffic participants who can use this information to support their decision making. To some extent, real-time traffic information is already available in many places. For example, in Singapore live traffic information is provided by Quantum Inventions (http://www.livetraffic.sg/). However, the live traffic information is currently still very crude and also lagging. Aggregating data from various sources to generate traffic information at a high resolution with a minimum of latency would represent a significant improvement over currently available services. The next step would be to use the aggregated data to initialize a simulation model of the traffic system in order to perform high-fidelity simulations that predict the traffic situation of the entire city in the near-future (e.g., 3-12 hours). An overview of the general information flow in the envisioned smart traffic system is illustrated in Figure 1. To summarize, the system contains three major components, data collection, modeling and simulation and participatory sensing. The 
mobile devices, along with other sources, provide data to the simulation engine which generates forecasts of traffic in real-time. Finally, these forecasts are provided back to the participants to aid them in their planning and routing.

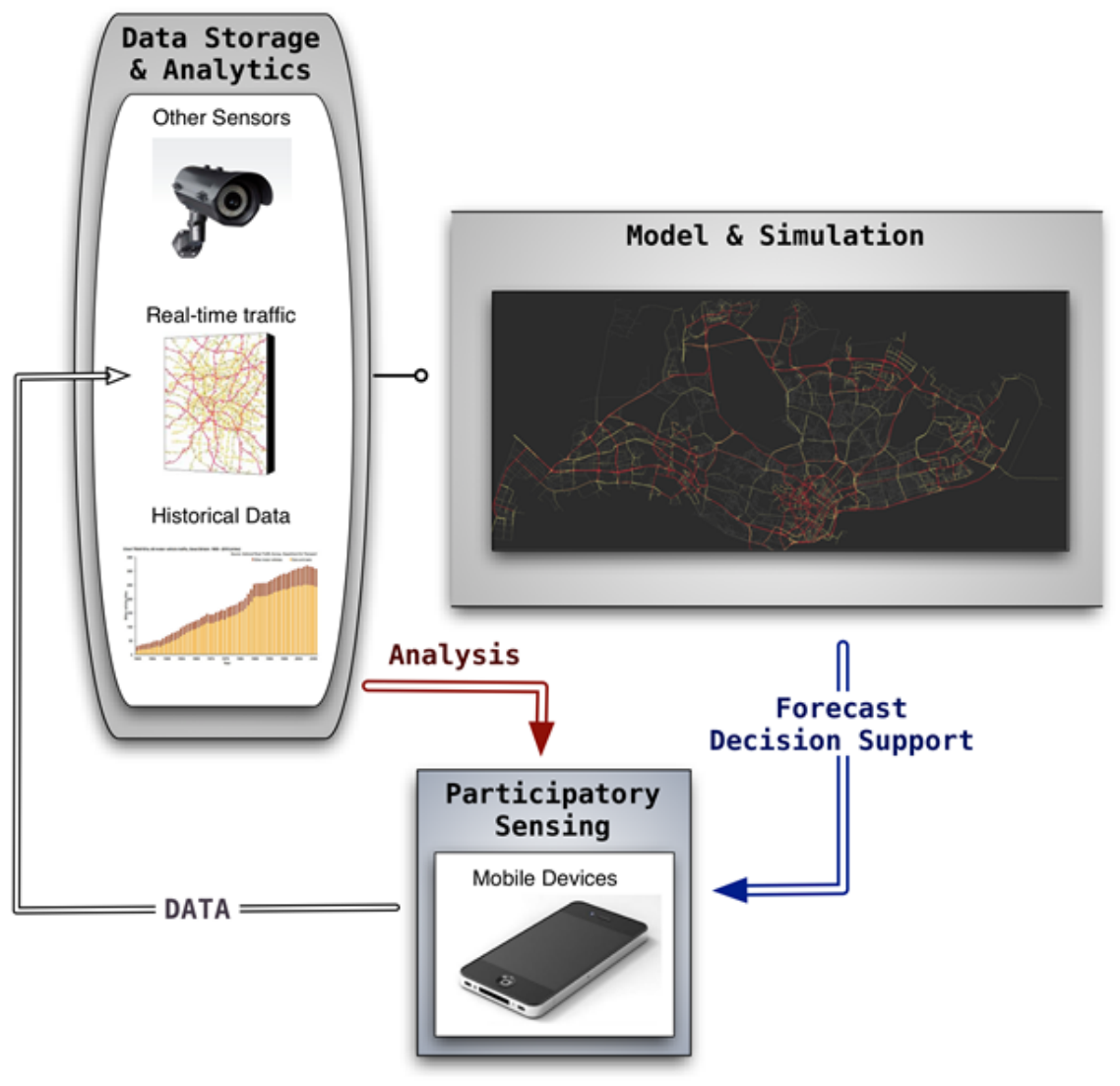

Figure 1: Overview of the general information flow in the envisioned real-time traffic monitoring and control system.

\subsection{Agent-based Traffic Simulation}

The real-time coupling of individual data to simulations imposes certain constraints on the form of modeling and simulation that can be used. Simulation methodologies that have explicit notions of the individual are necessary, in general these form individual-based models. Agent-based modeling is one form of such models, a method popularized by economists attempting to understand trader behavior, it has now been applied across many fields, from humans crowds, to biofilms, to traffic. Agent-based models consist of large numbers of heterogeneous, autonomous entities inhabiting a spatially explicit environment where macro level dynamics are said to emerge through the asynchronous interactions among these entities (Bonabeau 2002; Epstein 1999).

Traffic simulation is typically classified according to the level of detail at which they represent traffic flow. In terms of methodology there are macroscopic models, mesoscopic models and microscopic models, all of which lend themselves to different forms of problem, with varying levels of suitability. In macroscopic traffic simulation (Papageorgiou et al. 2010), traffic flow is modeled using fluid dynamics (Lighthill and Whitham 1955; Richards 1956) which analyzes the aggregate properties: speed, density, and flow, with respect to time and space. Further details are considered in mesoscopic traffic simulation (Ben-Akiva et al. 2010) which combines flow dynamics and simplified movement of vehicles (Barceló 2010). Microscopic 


\section{Aydt, Lees, and Knoll}

traffic simulation (Ben-Akiva et al. 2010) offers even more details and models individual driver-vehicle units and their movement within the road network. Detailed microscopic models require large amount of calibration data and vast computational resources, which makes large-scale microscopic traffic simulation challenging (Hollander and Liu 2008). Cellular automata (Nagel, Schreckenberg, and Others 1992) is one type of microscopic model that describes the movement of driver-vehicle units in a discrete, cellular space, with basic localized rules instead of complicated mathematical equations. While being microscopic, the simplicity of the rules and assumptions about the space make this approach less computationally intensive.

In the context of electro-mobility, we are currently working on an agent-based simulation framework which is specifically tailored to the simulation of an electric vehicle infrastructure: SEMSim (Scalable Electro-Mobility Simulator) (Xu, Aydt, and Lees 2012). This framework will support nanoscopic traffic simulations which is an extension of microscopic traffic simulation. In a nanoscopic traffic simulation, there are separate models for drivers and vehicles. This separation of driver and vehicle allows a high degree of flexibility. Firstly, it allows us to explicitly model and validate relevant driver behaviors and vehicle components independent from each other. Secondly, it makes it relatively easy to setup a heterogeneous population of agents. For example, given a variety of vehicle models, it is possible to reflect a particular distribution of different kinds of vehicles (e.g, electric vehicles models and combustion engine-based vehicle models). Similarly, a variety of driver characteristics can be used to reflect a particular distribution of behaviors. This is particularly useful, when considering the application of the simulator to different regions in the world. Since the concept of SEMSim is very modular, it is possible to replace any behavior or vehicle component model with one that is more suitable depending on the scope of the application. For example, driving behavior in different countries can be very different. This depends heavily, among other things, on cultural aspects. With SEMSim it is very easy to replace a typical German driving behavior model with a Singaporean driving behavior model, for instance.

\subsection{Degree of Participation}

With the rise of social networking it has become common practice to share all sorts of information with a selected few or even the general public. Although we expect that this trend will continue we cannot assume that we generally have all necessary (or rather desirable) information about traffic participants. Relevant information in this context refers to the position of traffic participants, their current speed, their current destinations, or even the routes they are planning to take in order to reach their destinations. Some, or all, of this information may not be available for modeling and simulation purposes. This can be due to either technical issues (e.g., not every vehicle is equipped with the necessary devices) or the unwillingness of people to share information for privacy purposes. Ideally, all real-world traffic participants will be represented by an individual agent in the agent-based traffic simulation. For modeling purposes, we therefore distinguish between black-box agents, gray-box agents, and white-box agents depending on the degree of participation.

A black-box agent does not share any information. The only way to determine the location of a black-box agent is by using external sensors (e.g., traffic camera). A gray-box agent shares information regarding its current position and speed (e.g., by means of a mobile phone or on-board navigation system). Finally, white-box agents share all relevant information, i.e., the same information that is shared by a gray-box agent plus information regarding the current destination and the chosen route. Sharing information about the destination and the chosen route can be done by a suitable on-board navigation system. Of course, there is still some uncertainty regarding whether a driver will actually follow the route suggested by the navigation system. However, it is reasonable to assume that most people will follow their navigation systems most of the time. Figure 2 illustrates the degree of participation of the different types of participants. 


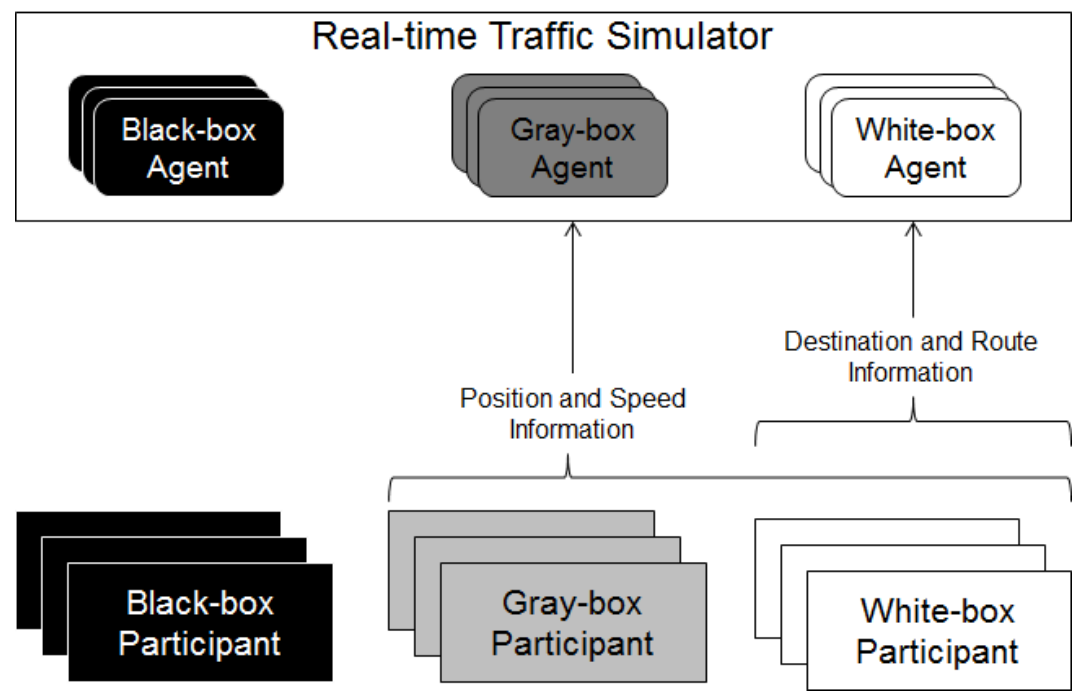

Figure 2: Illustration of the three degrees of participation: 1) black-box participants do not share any information; 2) gray-box participants share position and speed information; and 3) white-box agents share destination and route information in addition to the same information that is also shared by gray-box agents. Depending on the degree of their participation, real-word traffic participants are represented by corresponding agents in the simulation.

\section{SMART ROUTING APPLICATION}

\subsection{Global Route Optimization}

From the perspective of a smart routing service, white-box participants are the most important category of participants since they are willing to share information about their destination and routes. This information can be used by an appropriate server-based optimization engine to determine optimal routes for all white-box participants. This can be considered as an optimization problem with the objective to maximize the overall traffic throughput, minimize the overall travel times, or even to minimize the estimated energy consumption. In particular, the last objective is of great interest in the context of electro-mobility. In the real-world it is often necessary to consider multiple objectives in order to avoid over-optimization. It is also possible to consider various constraints. These constraints could be imposed by the preferences or limitations of the traffic participants. For example, such a constraint could impose a maximum route length for a path to the current destination. Such an upper bound could be motivated by individual preference but also by a limited range due to low battery charge.

Smart routing is initiated by a white-box traffic participant sending a route optimization request to the back-end server. This request contains a list of alternative routes that the traffic participant is willing to consider. It is up to the traffic participant to propose alternative routes that satisfy all important constraints. This is important as different traffic participants may have different constraints. In addition, by proposing the alternative routes, it is not necessary to disclose and further information to the back-end system (i.e., information about the constraints remain private). Another advantage is the simplification of the optimization process as it does not have to consider multiple constraints for all the various white-box traffic participants. In general, the optimization problem can be considered as a combinatorial problem. Since each white-box participant proposes a number of alternative routes, the problem becomes to identify the optimal combination of route choices for which the various global objectives can be achieved. The search space of this combinatorial problem is $\prod_{i=0}^{N-1} n_{i}$, where $n_{i}$ is the number of proposed alternative routes of the $i$-th agent and $N$ is the number of white-box agents in the system. It is not difficult to see that the size of the search space is growing exponentially with every additional white-box agent that wants 
to participate. Previous work has already addressed computational issues with large search spaces in the context of symbiotic simulation (Aydt et al. 2011). Similar techniques can also be applied in the case of smart routing.

Due to the complexity of the traffic system, it is difficult to anticipate the impact of different combinations of route choices on the overall traffic. For this purpose, every combination of route choices, further referred to as a what-if scenario, is evaluated by means of simulation. This simulation is based on the high-fidelity model which is continuously updated by using live data feeds. Each of the white-box participants is represented by an agent in the simulation. The various route choices of participants are used to setup the white-box agents in the simulation. The result of the simulation run is analyzed by calculating various performance values (e.g., average trip time for white-box agents, total energy consumption, etc.). Based on these performance values, it is possible to compare and rank different what-if scenarios. The primary goal of the optimization process is to identify a what-if scenario that optimizes the objectives. Since each what-if scenario has to be evaluated by using simulation, it is crucial to have the ability to simulate a large amount of what-if scenarios in a very short period of time. This can be achieved by using a high-performance computer (HPC). An overview of the smart routing service based on symbiotic simulation is illustrated in Figure 3.

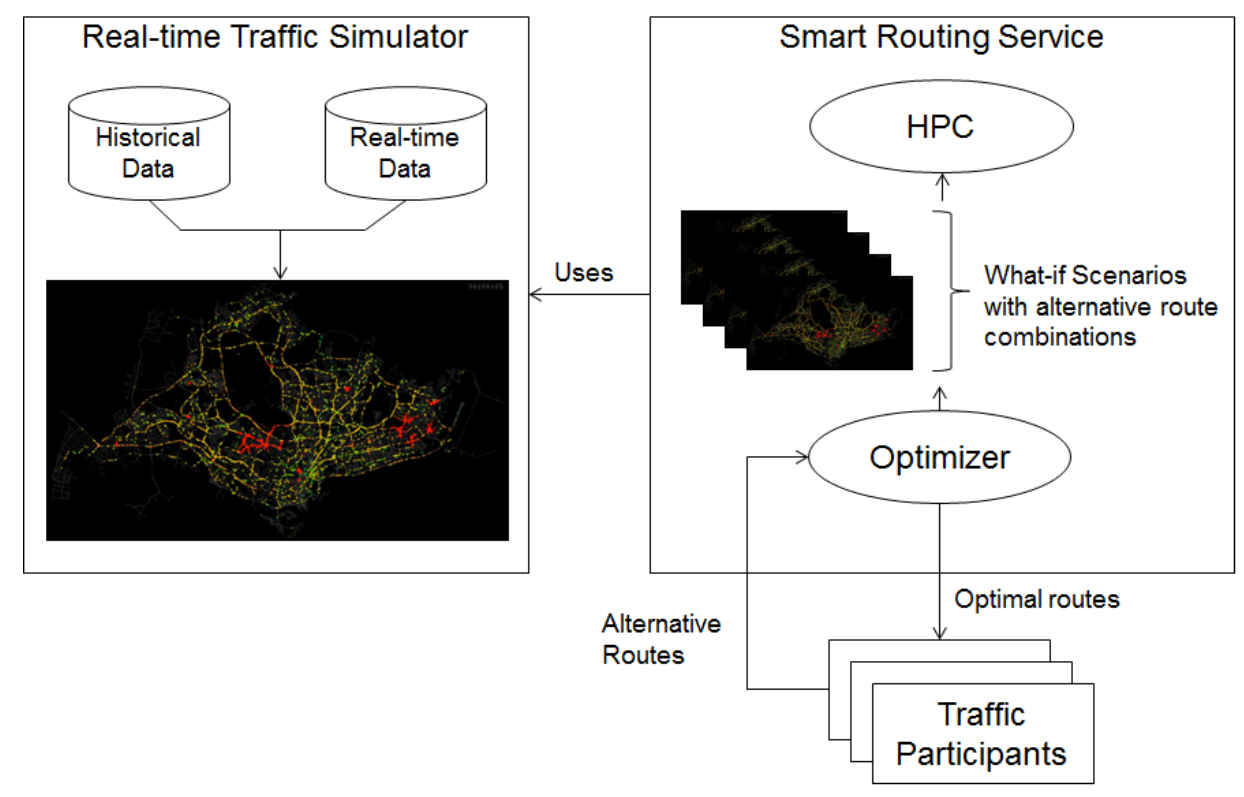

Figure 3: Overview of the smart routing service based on symbiotic simulation.

\subsection{Individual Route Optimization}

Traffic participants who do not want to share their current position and destination, can still benefit from the high-fidelity simulation forecasts. For example, based on the simulation results in a weighted graph of the road network can be generated where the weights represent the estimated traffic density of average speed of the road link. Figure 4 illustrates an example of the Singapore road network with color-coded information regarding the average speed of the traffic.

This information can be used by an on-board navigation system to determine a reasonably good route to the current destination. Systems with similar functionality already exist and are widely in use. For example, modern navigation systems are capable of incorporating real-time traffic data and information about traffic lights, for instance, to determine the fastest route. However, to the best of our knowledge none of these systems is leveraging on high-fidelity simulation-based forecasts such as the one described 


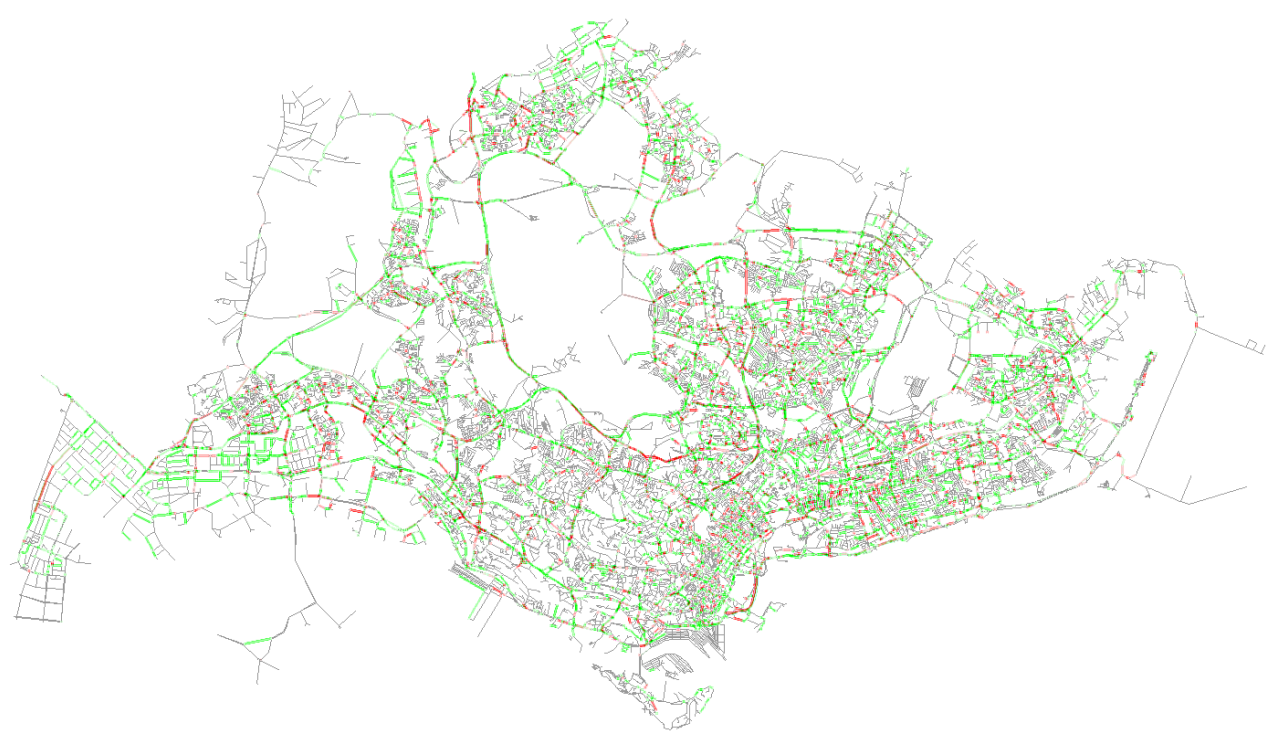

Figure 4: Example of simulation results that indicate the estimated average speed on the various road links of the Singapore road network. The colors green and red indicate that the average speed is expected to be faster or slower, respectively.

here. Therefore, traffic participants which are not willing to share information can still benefit from the simulation-based system.

\section{RESEARCH CHALLENGES}

In order to realize a symbiotic simulation-based smart traffic system, there are a number of challenges that need to be addressed. These challenges can be categorized into four groups: 1) sensitivity and consistency, 2) modeling and validation, 3) scalability and robustness, 4) participation.

\subsection{Sensitivity and Consistency}

A lot of data is required to perform high-fidelity simulations. While some of this data may be commercially available, other data may be proprietary and thus not available at all. Even if the data is available, it may not be available in sufficient quality or resolution. This raises concerns regarding the sensitivity of the simulation results with respect to uncertainties in the data that is used to initialize and drive a simulation. Another issue in this context is the sensitivity of the simulation due to temporal inconsistencies caused by latency for receiving data. This latency may be significant depending on the data source. It is important to analyze how sensitive the simulation is to several data-related inconsistencies. Furthermore, suitable methods have to be developed in order to deal with the various forms of inconsistencies. These methods should also be evaluated in order to see how different methods affect the outcome of simulation, i.e., to evaluate how sensitive the simulation is to these methods.

For the simulation-based smart traffic system to be effective, it is important to aggregate data, initialize the simulation, and execute a simulation in real-time. The notion of real-time here generally refers to seconds and minutes but cannot be strictly defined without a very specific use case in mind. For example, in case of an accident, a new forecast has to be created which incorporates information about this incident in order to make a prediction regarding how this situation will affect the traffic situation. In order for traffic participants to benefit from a revised forecast, the smart traffic system must have a reasonably short response time. In general, the faster an incident is reflected in the simulations, the faster the response time and thus the expected effectiveness of any service that leverages on the simulation system. Another form of 


\section{Aydt, Lees, and Knoll}

inconsistencies that need to be addressed are discrepancies between the traffic forecast (e.g., visualizations) for the traffic participants and the actual situation. For example, a traffic participant may experience a traffic jam due to an accident. However, this incident may not yet be reflected in the information provided by the smart traffic system.

\subsection{Modeling and Validation}

From a modeling perspective, the black-box agents are the most difficult to model as their respective traffic participants in the real world do not provide any information. Even information about their existence has to be inferred from various sensor data. Even if black-box agents can be identified, it is necessary to describe their behavior by using probabilistic models that have been created based on historical data and/or live data. Gray-box agents are initialized by using available position and speed data from traffic participants that are willing to share this information. However, due to inaccuracies in the GPS data, suitable algorithms may have to be employed in order to map the positions of the various traffic participants to the correct road. Similar to black-box agents, gray-box agents need to use probabilistic models for determining their route. White-box agents, on the other hand, can be initialized by not only using position and speed data but also by using available information about routes and destinations.

Since we intend to use nanoscopic traffic simulation, a general challenge is the modeling of vehicle components and driver behavior. In particular, driver behavior modeling is difficult as it involves creating models of the human decision making process. Black-box and gray-box agents, and to some extent also white-box agents, rely on driver behavior models which have to make up for the lack of data. If these models are not sufficiently accurate, the simulation results will not represent a good prediction of how traffic is going to develop in the next few hours. Depending on the overall degree of participation, there may be a significant number of black-box and gray-box agents. It is therefore necessary to develop appropriate behavior models and validate them within the scope of the application. Vehicle models are easier to develop and validate than behavior models since it is possible to collect a data and model the inner workings of vehicle components, such as air-conditioning and heating systems.

\subsection{Scalability and Robustness}

Nanoscopic traffic simulation is computationally even more expensive than microscopic traffic simulation. For this purpose, it is thus necessary to develop sophisticated methods that optimize the execution performance of a nanoscopic traffic simulation. We have described a distributed architecture for a scalable electromobility simulator (Xu, Aydt, and Lees 2012). The main challenge here is to design a simulator with good scalability in terms of numbers of vehicles. Given the computational requirements of a nanoscopic traffic simulation, it makes sense to think of ways to distribute and parallelize the simulation. Since we use an agent-based simulation, common practice would be to allocate agents to the various logical processes of the simulation. High performance can be achieved by minimizing the dependencies between the various logical processes of the simulation. Dependencies are caused by interactions between the various agents. Therefore, one possible approach to maintain high performance is to optimize the allocation of agents to logical processes. This is challenging because the optimal allocation of agents is continuously changing due to the dynamics of traffic.

A symbiotic simulation system is typically concerned with the evaluation of a large number of what-if scenarios in order to find a suitable solution to a given problem. This adds even more pressure on the performance of the simulator because if a real-time response is required, then a large number of what-if scenarios have to be evaluated in a very short period of time. For this purpose, it may be useful to employ meta-heuristics, such as evolutionary computing. While these methods do not necessarily find the global optimum, they can provide reasonably good solutions within a short period of time. When it comes to solving real-world problems, it is often more important to find a robust solution in time rather than finding the optimal solution too late or no solution at all. A robust solution is a solution that is effective in the real 


\section{Aydt, Lees, and Knoll}

world despite certain discrepancies between the simulation and reality caused by uncertainties in the model or data. In this context it should also be kept in mind that the definition of an optimum is problematic as the system is continuously changing and so does the optimum. Furthermore, in practice it is also often more important to find solutions that are robust and not necessarily optimal. This is particularly important for traffic systems, where robustness is crucial.

\subsection{Participation}

The effectiveness of the smart traffic system relies on the participation of traffic participants. Ideally, all participants provide all the desired information. However, making such assumptions is unrealistic - at least during an initial stage of the application. At a later stage, if the system has proven its usefulness, people may be more willing to provide the information as they can see a clear benefit from doing so. However, in the meantime, it is necessary to analyze the impact of degree of participation and evaluate the effectiveness of the traffic system even if participation is very low. At the same time, the privacy concerns need to be addressed. This may also include development of methods that may perform some of the computations on the side of the client which may reduce the amount of data that has to be send to the server. It may even be possible to perform a distributed simulation across all clients and thus resolving any privacy issues all together.

\section{SUMMARY AND FUTURE WORK}

Increasing the efficiency and robustness of traffic is an important issue for mega-cities. In future, this problem will become even more important. In this paper we have described our vision of a smart traffic system that leverages heavily on the concepts of living simulations and symbiotic simulation. This also involves computationally expensive simulation-based optimization, requiring the execution of many what-if simulations in a high-performance computing environment. This is distinctively different from existing work on simulation-based intelligent transportation systems (Fujimoto et al. 2006), for example, which aims at performing a distributed traffic simulation. Furthermore, our simulation will represent the traffic of an entire city with a very high resolution. This is different from existing simulators, such as DYNASMARTP or MATSim (http://www.matsim.org/), which often only simulate traffic on an aggregated level (i.e., macroscopic and mesoscopic traffic simulation). High resolution traffic simulation is computationally expensive. Our simulation engine will thus be specifically designed for parallel execution in an HPC environment (Xu, Aydt, and Lees 2012).

In this paper, we have described a smart routing application that would not only avoid limitations faced by currently used navigation systems, it would also enable traffic operators to further improve the traffic flow of a city by means of city-wide optimization. Furthermore, traffic operators would also have the possibility to steer the traffic on a city-scale by influencing the optimization engine. For example, this may be useful in case of big events that require to divert traffic in certain parts of the city. The living simulation and symbiotic simulation concepts require a lot of data from various sources. This data needs to be aggregated and incorporated into a simulation in real-time. This poses a variety of research challenges that need to be addressed in order to realize such a system. We have summarized the most important research issues which are concerned with 1) sensitivity and consistency, 2) modeling and validation, 3) scalability and robustness, and 4) participation. In our future work we will address these issues and perform various experiments that will demonstrate the effectiveness of our system.

\section{ACKNOWLEDGMENTS}

This work was financially supported by the Singapore National Research Foundation under its Campus for Research Excellence And Technological Enterprise (CREATE) programme. 
Aydt, Lees, and Knoll

\section{REFERENCES}

Aydt, H., W. Cai, S. Turner, and B. Gan. 2011, December. "Symbiotic Simulation for Optimisation of Tool Operations in Semiconductor Manufacturing". In Proceedings of the 2011 Winter Simulation Conference, edited by S. Jain, R. R. Creasey, J. Himmelspach, K. P. White, and M. Fu, 2088-2099. Piscataway, New Jersey: Institute of Electrical and Electronics Engineers, Inc.

Aydt, H., S. J. Turner, W. Cai, and M. Y. H. Low. 2008. "Symbiotic Simulation Systems: An Extended Definition Motivated by Symbiosis in Biology". In Proceedings of the 22nd Workshop on Principles of Advanced and Distributed Simulation, 109-116.

Barceló, J. 2010. "Models, Traffic Models, Simulation, and Traffic Simulation". In Fundamentals of Traffic Simulation, edited by J. Barceló, Volume 145 of International Series in Operations Research \& Management Science, 1-62. New York, NY: Springer New York.

Ben-Akiva, M., H. N. Koutsopoulos, C. Antoniou, and R. Balakrishna. 2010. "Traffic Simulation with DynaMIT”. In Fundamentals of Traffic Simulation, edited by J. Barceló, Volume 145 of International Series in Operations Research \& Management Science, 363-398. Springer New York.

Ben-Akiva, M., H. N. Koutsopoulos, T. Toledo, Q. Yang, C. F. Choudhury, C. Antoniou, R. Balakrishna, and J. Barceló. 2010. "Traffic Simulation with MITSIMLab". In Fundamentals of Traffic Simulation, edited by J. Barceló, Volume 145 of International Series in Operations Research \& Management Science, 233-268. New York, NY: Springer New York.

Bonabeau, E. 2002. "Agent-based modeling: Methods and techniques for simulating human systems". Proceedings of the National Academy of Sciences of the United States of America 99 (Suppl 3): $7280-7287$.

Epstein, J. M. 1999. “Agent-based computational models and generative social science”. Complexity 4 (5): 41-60.

Fujimoto, R., R. Guensler, M. Hunter, H. Kim, J. Lee, J. Leonard, M. Palekar, K. Schwan, and B. Seshasayee. 2006. "Dynamic data driven application simulation of surface transportation systems". Computational Science-ICCS 2006:425-432.

Fujimoto, R., D. Lunceford, E. Page, and A. M. Uhrmacher. 2002. "Grand Challenges for Modeling and Simulation: Dagstuhl report". Technical Report 350, Schloss Dagstuhl. Seminar No 02351.

Gerlough, D. L. 1956. "Simulation of Freeway Traffic by an Electronic Computer". Highway Research Board Proceedings 35:543 - 547.

Bengt Halvorson 2011. "2012 Mitsubishi i: First Drive, U.S.-Spec MiEV”. http://www.greencarreports. com/news/1066863_2012-mitsubishi-i-first-drive-u-s--spec-miev. Last retrieved: 2012-05-22.

Hollander, Y., and R. Liu. 2008, January. "The principles of calibrating traffic microsimulation models". Transportation 35 (3): 347-362.

Lighthill, M. J., and G. B. Whitham. 1955, May. "On Kinematic Waves. II. A Theory of Traffic Flow on Long Crowded Roads". Proceedings of the Royal Society A: Mathematical, Physical and Engineering Sciences 229 (1178): 317-345.

Nagel, K., M. Schreckenberg, and Others. 1992. "A cellular automaton model for freeway traffic". Journal de Physique I 2 (12): 2221-2229.

Papageorgiou, M., I. Papamichail, A. Messmer, and Y. Wang. 2010. "Traffic Simulation with METANET". In Fundamentals of Traffic Simulation, edited by J. Barceló, Volume 145 of International Series in Operations Research \& Management Science, 399-430. New York, NY: Springer New York.

Richards, P. I. 1956. "Shock Waves on the Highway". Operations Research 4 (1): 42-51.

Wilson Rothman 2011. “'Death by GPS': Could it happen to you?”. http://www.technolog.msnbc.msn. com/technology/technolog/death-gps-could-it-happen-you-125321. Last retrieved: 2012-05-22.

Snoonian, D. 2003. "Smart buildings". Spectrum, IEEE 40 (8): 18 - 23.

Turrentine, T., D. Garas, A. Lentz, and J. Woodjack. 2011, May. "The UC Davis MINI E Consumer Study". Technical Report UCD-ITS-RR-11-05, Institute of Transportation Studies, University of California, Davis. 
Wang, F.-Y., D. Zeng, and L. Yang. 2006. "Smart Cars on Smart Roads: An IEEE Intelligent Transportation Systems Society Update". Pervasive Computing, IEEE 5 (4): 68 - 69.

$\mathrm{Xu}, \mathrm{Y} ., \mathrm{H}$. Aydt, and M. Lees. 2012. "SEMSim: A Distributed Architecture for Multi-scale Traffic Simulation". In Proceedings of the 26th ACM/IEEE/SCS Workshop on Principles of Advanced and Distributed Simulation (to appear).

\section{AUTHOR BIOGRAPHIES}

HEIKO AYDT is a Research Fellow at TUM CREATE Ltd., a company dedicated to research in all aspects of electro-mobility. He received his PhD degree in Computer Science from Nanyang Technological University (NTU) in Singapore and his MSc degree from the Royal Institute of Technology (KTH) in Stockholm. His research interests are agent-based simulation, simulation-based optimization, and evolutionary computing. His email address is heiko.aydt@tum-create.edu.sg.

MICHAEL HAROLD LEES is an Assistant Professor in the School of Computer Engineering, Nanyang Technological University (NTU). Dr Lees received his PhD from the School of Computer Science at the University of Nottingham, UK. He has post-doctoral experience at NTU, the University of Nottingham University and the University of Birmingham, UK. His research interests are primarily in modeling and simulation of large scale complex systems, he is particularly interested in understanding the effect that human behavior has on such systems and the important role that individual behavioral interactions have on system level dynamics. He is currently workshop chair of the International Conference on Computational Science (ICCS) and an editor of the Journal of Computational Science (JOCS). His email address is mhlees@ntu.edu.sg.

ALOIS KNOLL received his diploma (MSc) degree in Electrical/Communications Engineering from the University of Stuttgart and his PhD degree in Computer Science from the Technical University of Berlin. He served on the faculty of the computer science department of TU Berlin until 1993, when he qualified for teaching computer science at a university (habilitation). He then joined the Technical Faculty of the University of Bielefeld, where he was a full professor and the director of the research group Technical Informatics until 2001. Between May 2001 and April 2004 he was a member of the board of directors of the Fraunhofer-Institute for Autonomous Intelligent Systems. At AIS he was head of the research group "Robotics Construction Kits", dedicated to research and development in the area of educational robotics. Since autumn 2001 he has been a professor of Computer Science at the Computer Science Department of the Technische Universitt München. He is also on the board of directors of the Central Institute of Medical Technology at TUM (IMETUM-Garching); between April 2004 and March 2006 he was Executive Director of the Institute of Computer Science at TUM. His research interests include cognitive, medical and sensor-based robotics, multi-agent systems, data fusion, adaptive systems and multimedia information retrieval. His email address is knoll@in.tum.de. 